\section{Author Correction: A CRISPR/Cas system mediates bacterial innate immune evasion and virulence}

Timothy R. Sampson, Sunil D. Saroj, Anna C. Llewellyn, Yih-Ling Tzeng \& David S. Weiss

Correction to: Nature https://doi.org/10.1038/nature12048, published online 14 April 2013.

We have been unable to reproduce the RNA stability experiment in Fig. 2a of this Letter, and no longer observe a Cas9-dependent decrease in the relative levels of the FTN_1103 transcript. The new data reveal that the wild-type and cas 9 deletion mutant $(\Delta$ cas 9$)$ strains have equivalent levels of FTN_1103 mRNA at each time point (see Fig. 1 of this Amendment). We do not have an explanation for the discrepancy with the original data, but it may in part be due to the complications derived from measuring the stability of the FTN_1103 transcript in strains with very different baseline levels of this mRNA. We have also been unable to replicate the results of the immunoprecipitation experiments in Fig. $2 \mathrm{~g}$ of the original Letter, and no longer observe lower levels of the FTN_1103 transcript after immunoprecipitation with Cas9(R59A)-Flag as compared with wild-type Cas9-Flag (see Fig. 2 of this Amendment). We do still observe the original trend of higher levels of small, CRISPR-Cas-associated RNA (scaRNA) and transactivating crRNA (tracrRNA) in the wild-type Cas9-Flag immunoprecipitation as compared with the Cas9(R59A)-Flag mutant (Fig. 2e, $\mathrm{f}$ of the original Letter). However, we cannot rule out that these new results are simply a reflection of the overall levels of these transcripts in the different strains, owing to the biological lack of a Cas9-regulated control outside the FTN_1103 locus. Independently of the immunoprecipitation experiment, there remain robust genetic data indicating that scaRNA and tracrRNA are required for Cas9-dependent repression of FTN_1103 mRNA levels.

In addition, in Fig. $2 \mathrm{~h}$ of this Letter, a tracrRNA mutant (tracrR$\mathrm{NA}(\mathrm{rc} 13-17)$ ) containing substitutions in the putative interaction site with FTN_1103 was used to suggest that the expression of FTN_1103 was repressed via interaction with the tracrRNA. Although not known at the time, subsequent crystallographic data ${ }^{1}$ indicate that the mutation to tracrRNA was in an important stem-loop region that interacts with Cas9, and Cas9 has been shown to be required for stable tracrRNA levels ${ }^{2}$. For these reasons, we can no longer definitively deduce the role of the mutated tracrRNA bases in the repression of FTN_1103 and have no evidence to support a role of tracrRNA in direct interaction with FTN_1103 RNA.

Together, these results suggest that we do not have evidence to support RNA degradation as the mechanism that underlies Cas9mediated regulation of FTN_1103 mRNA expression. The other experiments and conclusions presented in the Letter are unaffected by this Amendment, and we apologize for the previous misleading data and the effect that they may have had on others in the field. We are grateful to Hannah K. Ratner for identifying these errors, conducting the experiments described here along with Siddharth Jaggavarapu, and for writing the majority of this Amendment. The original Letter has not been corrected.

1. Deltcheva, E. et al. CRISPR RNA maturation by trans-encoded small RNA and host factor RNase III. Nature 471, 602-607 (2011).

2. Hirano, H. et al. Structure and engineering of Francisella novicida Cas9. Cell 164, 950-961 (2016).

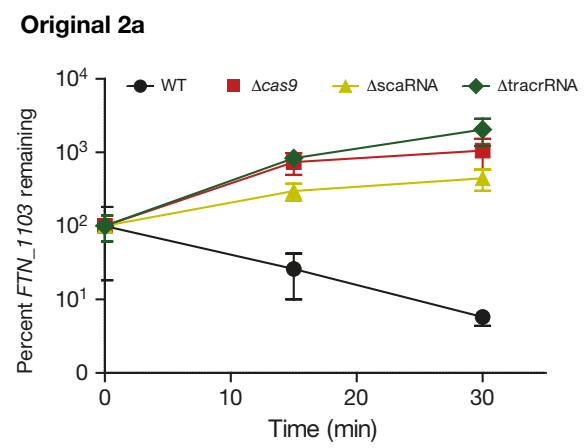

Fig. 1 | The incorrect published Fig. 2a and the corrected Fig. 2a of the original Letter. Stability of FTN_1103 RNA in wild-type (WT) and $\Delta$ cas 9 Francisella novicida. Levels of FTN_1103 RNA (relative to $u v r D$ ) were measured by quantitative PCR (qPCR) at 0,15 and

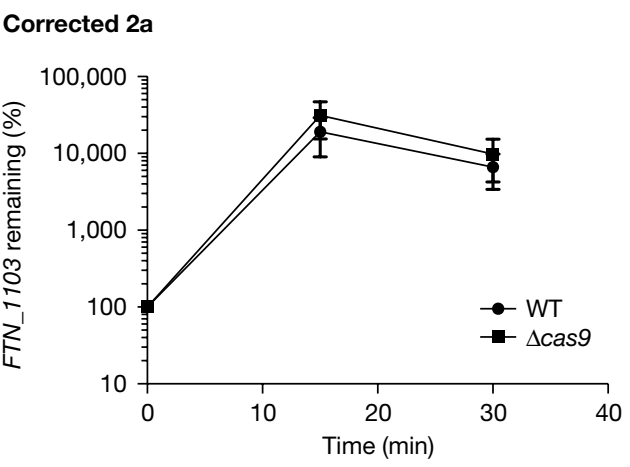

30 min after treatment with rifampicin. Results are indicated as the percentage of FTN_1103 mRNA remaining from the levels at $t=0$ $(n=8)$. 

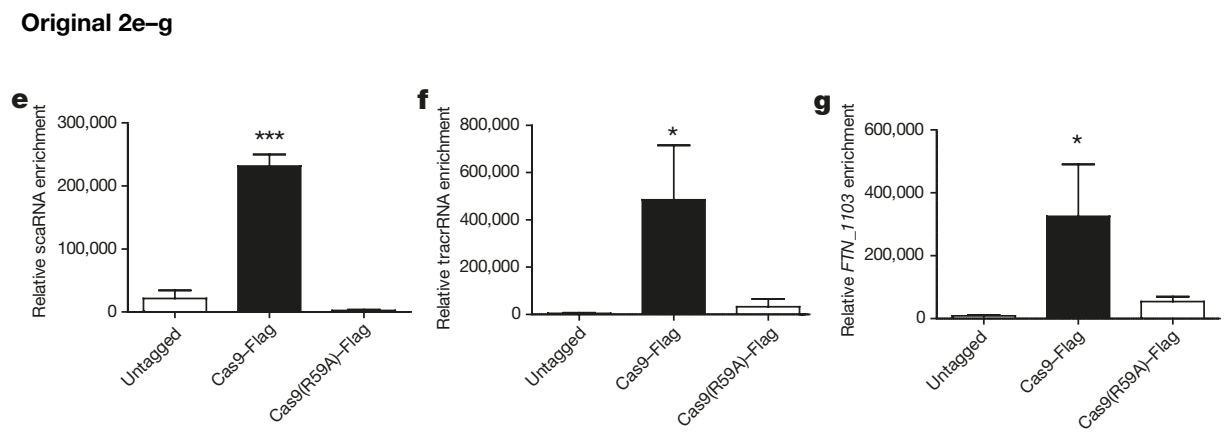

\section{Corrected 2e-g}
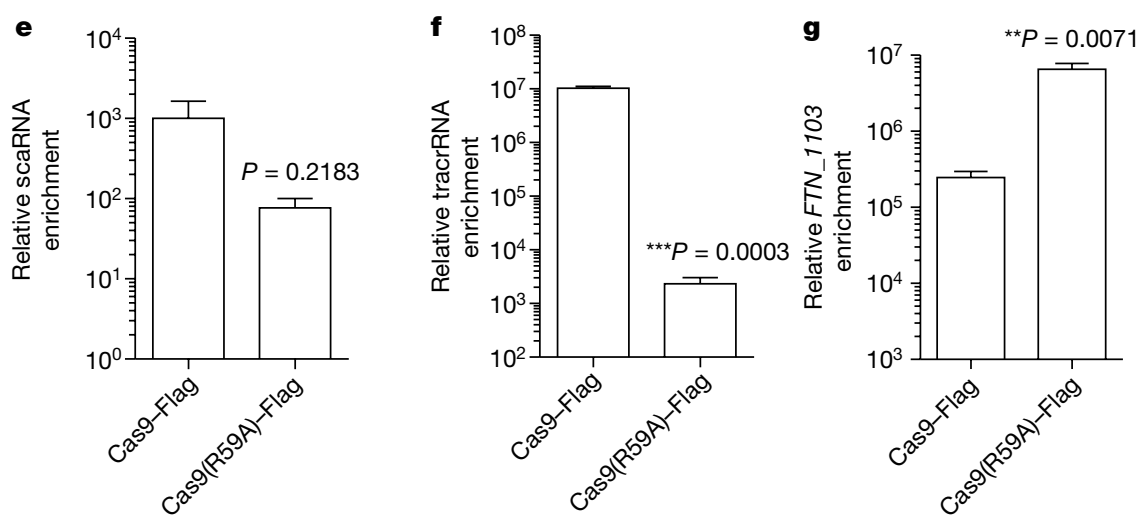

Fig. $2 \mid$ The incorrect published Fig. $2 \mathrm{e}-\mathrm{g}$ and the corrected Fig. $2 \mathrm{e}-\mathrm{g}$ of the original Letter. e-g, qPCR after immunoprecipitation of

Levels of scaRNA (e), tracrRNA (f) and FTN_1103 (g) were wild-type Cas9-Flag or mutant Cas9(R59A)-Flag from cell lysates. quantified by qPCR relative to $u v r D(n=3)$. Data are mean and s.e.m. $* * P \leq 0.005$; $* * * P \leq 0.001$, two-tailed Student's $t$-test. 